\title{
A NOTE ON A THEOREM OF HARDY ON FOURIER CONSTANTS
}

\section{RICHARD BELLMAN}

In Note LXVI of his Notes on some points in the integral calculus $[1],{ }^{1}$ Hardy proves the following theorem.

TheOREM 1 (HARdy). If $a_{1}, a_{2}, \cdots, a_{n}, \cdots$ are the Fourier constants of a function of $L^{p}, p \geqq 1$, then $A_{1}, A_{2}, \cdots, A_{n}, \cdots$ are also the Fourier constants of a function of $L^{p}$, where $A_{n}=n^{-1} \sum_{1}^{n} a_{k}$.

Hardy restricts the class of functions considered to even functions, with mean value over a period zero, and the same restriction shall be observed here.

We wish to prove a "dual" to Theorem 1, namely:

THEOREM 2. If $b_{1}, b_{2}, \cdots, b_{n}, \cdots$ are the Fourier constants of $a$ function, $g(x)$, of $L^{p}, p>1$, then $B_{1}, B_{2}, \cdots, B_{n}, \cdots$ are also the Fourier constants of a function, $G(x)$, of $L^{p}$, where $B_{n}=\sum_{n}^{\infty} b_{k} k^{-1}$, and if $f(x) \sim \sum a_{n} \cos n x$ is any function of $L^{p^{\prime}}, F(x) \sim \sum_{1}^{\infty} A_{n} \cos n x$, then, $1 / p+1 / p^{\prime}=1$,

$$
\int_{0}^{\pi} f(x) G(x) d x+\int_{0}^{\pi} F(x) g(x) d x=0 .
$$

There is a difference between the theorems. The case $p=1$ is omitted in Theorem 2, and necessarily, since $g(x) \sim \sum_{n=2}^{\infty} \cos n x / \log n$ belongs to $L$, but the corresponding $B_{n}$ do not even exist.

Hardy's method depends upon an explicit representation of the function $F(x)$ in terms of $f(x)$. In the case of the theorem to be proven, this representation does not seem to facilitate matters. The method used will depend upon some general theorems on Fourier series, together with the original theorem of Hardy. For the case $p=2$, the proof is immediate, since it can be shown that the convergence of $b_{n}^{2}$ implies the convergence of $B_{n}^{2}$ (this result is also due to Hardy, and was the origin of this type of theorem). In particular, this series converges for a function belonging to $L^{p}$, for $p \geqq 2$, and thus there exists a function of $L^{2}$, having the $B_{n}$ as Fourier coefficients.

The method of proof depends upon the observation that, purely formally, partial summation yields

Received by the editors January 28, 1944.

1 Numbers in brackets refer to the references cited at the end of the paper. 


$$
\sum_{1}^{\infty} a_{n} B_{n}=-\sum_{1}^{\infty} A_{n} b_{n} .
$$

The justification of this will constitute the principal part of the proof. Three lemmas will be required.

Lemma 1 [2]. A necessary and sufficient condition that the series $\sum_{1}^{\infty} a_{n} \cos n x$ should be a Fourier series of a function of $L^{p}, p>1$, is that for every $g(x)$ belonging to $L^{p^{\prime}}\left(1 / p+1 / p^{\prime}=1\right)$ with Fourier coefficients $a_{n}^{\prime}$, the series $\sum_{1}^{\infty} a_{n} a_{n}^{\prime}$ should be finite $(C, 1)$.

A series is finite $(C, 1)$ when the arithmetic mean of its partial sums is bounded.

LEMMA 2. If $H(x) \sim \sum_{1}^{\infty} c_{n} \cos n x$ is a function of $L^{p}, p>1$, then

$$
\left|\sum_{1}^{N} c_{n}\right|=O\left(N^{1 / p}\right)
$$

Proof of Lemma 2.

$$
\sum_{1}^{N} c_{n}=\frac{1}{\pi} \int_{0}^{\pi} H(x)\left[\sum_{1}^{N} \cos n x\right] d x .
$$

Using Hölder's inequality

$$
\begin{aligned}
\left|\sum_{1}^{N} c_{n}\right| & \leqq \frac{1}{\pi}\left[\int_{0}^{\pi}|H(x)| p d x\right]^{1 / p}\left[\int_{0}^{\pi}\left|\sum_{1}^{N} \cos n x\right|^{p^{\prime}} d x\right]^{1 / p^{\prime}} \\
& =O\left[\int_{0}^{\pi}\left|\sum_{1}^{N} \cos n x\right|^{p^{\prime}} d x\right]^{1 / p^{\prime}} \\
& =O\left[\int_{0}^{\pi}\left|\frac{\sin N x}{x}\right|^{p^{\prime}} d x\right]^{1 / p^{\prime}}=O\left(N^{1 / p}\right) .
\end{aligned}
$$

This finishes the proof of the lemma.

LeMma $3 .^{2}$ If $H(x) \sim \sum_{1}^{n} c_{n} \cos n x$ belongs to $L^{p^{\prime}}, p>1$, then

$$
\left|\sum_{N}^{\infty} \frac{c_{n}}{n}\right|=O\left(N^{-1 / p}\right) \text {. }
$$

Proof of Lemma 3. Let $\sum_{1}^{\infty} c_{\nu}=s_{n}$, then

$$
\sum_{n}^{m} \frac{c_{\nu}}{\nu}=m^{-1} s_{m}-n^{-1} s_{n-1}+\sum_{n}^{m-1} \frac{s_{\nu}}{\nu(\nu+1)} .
$$

${ }^{2}$ The above proof of Lemma 3 and a part of the proof of Theorem 2 are due to Professor O. Szász and are simpler and more elegant than the original. 
By Lemma 2, $s_{m}=O\left(m^{1 / p^{\prime}}\right)$, hence $m^{-1} s_{m} \rightarrow 0$, and

$$
\begin{aligned}
\sum_{n}^{\infty} \nu^{-1} c_{\nu} & =\frac{-s_{n-1}}{n}+\sum_{n}^{\infty} \frac{s_{\nu}}{\nu(\nu+1)} \\
& =O\left(n^{1 / p^{\prime}-1}\right)+O\left(\sum_{n}^{\infty} \nu^{1 / p^{\prime}-2}\right) \\
& =O\left(n^{1 / p^{\prime}-1}\right)=O\left(n^{-1 / p}\right) .
\end{aligned}
$$

Proof of Theorem 2. $A_{n}$ and $B_{n}, f(x), g(x), F(x), G(x)$ are defined as in Theorems 1 and 2 . We divide the proof into three parts. The first part will consist of showing that $G(x)$ belongs to $L^{p}, p>1$, whenever $g(x)$ does. In virtue of Lemma 1 , it is sufficient to show

$$
\sum_{1}^{n} B_{\nu} a_{\nu}=O(1) \quad \text { as } n \rightarrow \infty,
$$

where $g(x) \sim \sum b_{n} \cos n x$ is a function of $L^{p}$, and $f(x) \sim \sum a_{n} \cos n x$ is a function of $L^{p^{\prime}}$.

By partial summation

$$
\sum_{1}^{n} B_{\nu} a_{\nu}=B_{n} \sum_{1}^{n} a_{\nu}-\sum_{1}^{n-1} A_{\nu} b_{\nu}
$$

Using Theorem 1, $F(x) \sim \sum A_{n} \cos n x$ belongs to $L^{p^{\prime}}$, and thus, using the generalized Parseval theorem, $\sum_{1}^{\infty} A_{\nu} b_{\nu}$ is convergent. Furthermore, $\sum_{1}^{n} a_{\nu}=O\left(n^{1 / p^{\prime}}\right)$, by Lemma 2 , and $B_{r}=O\left(n^{-1 / p^{\prime}}\right)$, by Lemma 3 . Hence

$$
B_{n} \sum_{1}^{n} a_{\nu}=O\left(n^{1 / p^{\prime}}\right) O\left(n^{-1 / p^{\prime}}\right)=O(1),
$$

and thus $G(x)$ belongs to $L^{p}$.

The second part consists of showing that $\sum_{1}^{\infty} B_{\nu} a_{\nu}$ converges. This is immediate by the generalized Parseval theorem (Hausdorff-Young theorem) since $G(x)$ belongs to $L^{p}, f(x)$ to $L^{p^{\prime}}$.

The third part consists of showing that $\sum_{1}^{\infty} A_{n} b_{n}=-\sum_{1}^{\infty} a_{n} B_{n}$ and using (1) this is true if, and only if, $B_{n} \sum_{1}^{\infty} a_{\nu}=o(1)$.

We have to consider two cases.

Case 1. $f(x)$ belongs to $L^{p}, 1<p \leqq 2$. Then, by the Hausdorff-Young theorem, $\sum\left|a_{n}\right|^{p^{\prime}}$ converges, and thus

$$
\left|\sum_{1}^{N} a_{n}\right|=o\left(N^{1 / p}\right) .
$$

Case 2. $f(x)$ belongs to $L^{p}, p>2$, and thus $g(x)$ belongs to $L^{p^{\prime}}$, 
$1<p^{\prime} \leqq 2$. Then, as before, $\sum\left|b_{n}\right|^{p}$ converges, and this entails

$$
\left|\sum_{N}^{\infty} \frac{b_{n}}{n}\right|=o\left(N^{-1 / p}\right)
$$

Thus for all $p>1$, .

$$
\left|B_{n} \sum_{1}^{n} a_{\nu}\right|=\left\{\begin{array}{l}
o\left(N^{1 / p}\right) O\left(N^{-1 / p}\right) \\
O\left(N^{-1 / p}\right) o\left(N^{1 / p}\right)
\end{array}\right\}=o(1) .
$$

This finishes the proof of Theorem 2.

It is interesting to see how these results arise naturally in dealing with Fourier integrals. The processes will be purely formal, although they could be justified by similar methods.

$$
\begin{aligned}
f(t) & =\int_{0}^{\infty} F(x) \cos x t d x \\
\int_{0}^{t} f(t) d t & =\int_{0}^{\infty} F(x) \frac{\sin x t}{x} d x=t \int_{0}^{\infty}\left(\int_{x}^{\infty} \frac{F(u)}{u} d u\right) \cos x t d x .
\end{aligned}
$$

The last result is obtained by integrating by parts, assuming that the integrated term drops out. Thus

$$
\frac{1}{t} \int_{0}^{t} f(t) d t=\int_{0}^{\infty}\left(\int_{x}^{\infty} \frac{F(u)}{u} d u\right) \cos x t d x .
$$

Similarly

$$
\begin{aligned}
\int_{t}^{\infty} \frac{f(t)}{t} d t & =\int_{0}^{\infty} F(x)\left[\int_{t}^{\infty} \frac{\cos x t}{t}\right] d x \\
& =\int_{0}^{\infty} F(x)\left[\int_{x t}^{\infty} \frac{\cos u}{u} d u\right] d x .
\end{aligned}
$$

Integrating the last expression by parts, and assuming the integrated part vanishes, there results

$$
\int_{t}^{\infty} \frac{f(t)}{t} d t=\int_{0}^{\infty}\left[\frac{1}{x} \int_{0}^{x} F(u) d u\right] \cos x t d x .
$$

1. G. H. Hardy, Notes on some points in the integral calculus, Mesesnger of Mathematics vol. 58 (1928) pp. 50-52.

2. A. Zygmund, Trigonometrical series, Warsaw, 1935, 4.63, Lemma, p. 103.

Princeton University 